\title{
Bezier Curves for Solving Fredholm Integral Equations of the Second Kind
}

\author{
F. Ghomanjani, ${ }^{1}$ M. H. Farahi, ${ }^{1,2}$ and A. Kılıçman ${ }^{3}$ \\ ${ }^{1}$ Department of Mathematics, Ferdowsi University of Mashhad, Mashhad, Iran \\ ${ }^{2}$ The Center of Excellence on Modelling and Control Systems (CEMCS), Mashhad, Iran \\ ${ }^{3}$ Department of Mathematics and Institute for Mathematical Research, Universiti Putra Malaysia, \\ 43400 Serdang, Selangor, Malaysia
}

Correspondence should be addressed to A. K1lıçman; kilicman@yahoo.com

Received 10 July 2013; Accepted 11 October 2013; Published 5 January 2014

Academic Editor: Fazal M. Mahomed

Copyright (C) 2014 F. Ghomanjani et al. This is an open access article distributed under the Creative Commons Attribution License, which permits unrestricted use, distribution, and reproduction in any medium, provided the original work is properly cited.

The Bezier curves are presented to estimate the solution of the linear Fredholm integral equation of the second kind. A direct algorithm for solving this problem is given. We have chosen the Bezier curves as piecewise polynomials of degree $n$ and determine Bezier curves on $[0,1]$ by $n+1$ control points. Numerical examples illustrate that the algorithm is applicable and very easy to use.

\section{Introduction}

Integral equations are often involved in the mathematical formulation of physical phenomena, and they can be encountered in various fields of science such as physics [1], biology [2], and engineering (see $[3,4]$ ). But we can also use it in numerous applications, such as control, biomechanics, elasticity, economics, electrical engineering, electrodynamics, electrostatics, fluid dynamics, game theory, heat and mass transfer, medicine, oscillation theory, plasticity, and queuing theory [5]. Fredholm integral equations of the second kind are shown in studies which include airfoil theory [6], elastic contact problems (see $[7,8]$ ), fracture mechanics [9], combined infrared radiation, and molecular conduction [10]. Many different basic functions have been used to estimate the solution of integral equations, such as orthogonal functions and wavelets (see [11, 12]). Depending on the structure, the orthogonal functions may be widely classified into three families [13]. The first includes sets of piecewise constant orthogonal functions (e.g., Walsh, blockpulse, Haar, etc.). The second consists of sets of orthogonal polynomials (e.g., Laguerre, Legendre, Chebyshev, etc.). The third are the widely used sets of sine-cosine functions in the Fourier series. Fredholm integral equations of the second kind are much more difficult to solve than ordinary differential equations. Therefore, many authors have tried various transform methods to overcome these difficulties (see $[11,12])$. Recently, hybrid functions have been applied extensively for solving differential equations or systems, and they proved to be a useful mathematical tool. The pioneering work in system analysis via hybrid functions was led in [14, 15], who first derived an operational matrix for the integrals of the hybrid function vector and paved the way for the hybrid function analysis of the dynamic systems. But they derived the matrix of small order, and the calculations are not enough to achieve high accuracy. Hsiao [16] presented the properties of hybrid functions which consist of blockpulse functions plus the Legendre polynomials. Based upon some useful properties of hybrid functions, integration of the cross product, a special product matrix and a related coefficient matrix with optimal order are applied to solve these integral equations. The main characteristic of this technique is to convert an integral equation into an algebraic one. Maleknejad and Mahmoudi [17] used a simple base, a combination of block-pulse functions on $[0,1]$ and the Taylor polynomials, that is called the hybrid Taylor block-pulse functions, to solve the linear Fredholm integral equation of the second kind. One of the advantages of this method is that the coefficients of expansion of each function in this base could be computed directly without estimation. 
Consider the following integral equation:

$$
y(t)=\int_{0}^{1} k(t, s) y(s) d s+x(t)
$$

where $x(t) \in L^{2}[0,1), k(t, s) \in L^{2}([0,1) \times[0,1)), y(t)$ is an unknown function.

In this paper, we discuss a technique similar to that used in [18] for solving integral equations by using the Bezier control points. There are many papers and books that deal with the Bezier curves or surface techniques. Harada and Nakamae [19] and Nürnberger and Zeilfelder [20] used the Bezier control points in approximated data and functions. Zheng et al. [21] proposed the use of the control points of the Bernstein-Bezier form for solving differential equations numerically, and also Evrenosoglu and Somali [18] used this approach for solving singular-perturbed two-point boundary value problems. The Bezier curves are used in solving partial differential equations; besides, Wave and Heat equations are solved in Bezier form (see [22-25] ). Wu [26] presented the least squares method for solving partial differential equations on arbitrary polygon domain by the Bezier control points. $\mathrm{Wu}$ [26] used triangular Bezier patches of degree $n$ with $C^{k}$ continuity to approximate the exact solution of partial differential equations. Bezier curves are used for solving dynamical systems (see [27]), also the Bezier control points method is used for solving delay differential equation (see [28]). Some other applications of the Bezier functions and control points are found in ([29-31]), that are used in computer-aided geometric design and image compression.

The use of the Bezier curves for solving Fredholm integral equations of the second kind is a novel idea. Although the method is very easy to be used and straightforward, the obtained results are satisfactory (see the numerical results).

We suggest a technique similar to that used in [28] for solving Fredholm integral equations of the second kind. The current paper is organized as follows.

Presented algorithm will be stated in Section 2. In Section 3, the convergence analysis will be presented. Some numerical examples are solved in Section 4 which show the efficiency and reliability of the method. Finally, Section 5 will give a conclusion in brief.

\section{The Algorithm}

Our strategy is to use Bezier curves to approximate the solutions $y(t)$ by $v(t)$ where $v(t)$ is given below. Define the Bezier polynomial of degree $n$ that approximates the values of $y(t)$ over the interval $\left[t_{0}, t_{f}\right]$ as follows:

$$
v(t)=\sum_{r=0}^{n} a_{r} B_{r, n}\left(\frac{t-t_{0}}{h}\right)
$$

where $h=t_{f}-t_{0}$

$$
B_{r, n}\left(\frac{t-t_{0}}{h}\right)=\left(\begin{array}{l}
n \\
r
\end{array}\right) \frac{1}{h^{n}}\left(t_{f}-t\right)^{n-r}\left(t-t_{0}\right)^{r}
$$

is the Bernstein polynomial of degree $n$ over the interval $\left[t_{0}, t_{f}\right]$, and $a_{r}$ is the control point (see [21]). By substituting (2) in (1), one may define $R_{1}(t)$ for $t \in\left[t_{0}, t_{f}\right]$ as follows:

$$
R_{1}(t)=v(t)-\left(\int_{t_{0}}^{t_{f}} k(t, s) v(s) d s+x(t)\right) .
$$

In Section 3, the convergence of this method is proven by Bezier curves when the degree of the approximate solution, $n$, tends to infinity.

Now, we define the residual function over the interval $\left[t_{0}, t_{f}\right]$ as follows:

$$
R=\int_{t_{0}}^{t_{f}}\left(\left\|R_{1}(t)\right\|^{2}\right) d t
$$

where $\|\cdot\|$ is the Euclidean norm. Our aim is to solve the following problem over the interval $\left[t_{0}, t_{f}\right]$ :

$$
\begin{aligned}
& \min \\
& \text { s.t. }
\end{aligned}
$$

When the minimization problem (6) is posed, the condition $v\left(t_{0}\right)=v_{0}$ is equivalent to fix the first control point $a_{0}=v_{0}$. The mathematical programming problem (6) can be solved by many subroutine algorithms, and we used Maple 12 to solve this optimization problem.

\section{Convergence Analysis}

In this section without the loss of generality, we analyze the convergence of the control-point-based method when applied to the integral equation (1) with the time interval $[0,1]$. So, the following problem is considered:

$$
\begin{gathered}
L(t, y(t))=y(t)-\int_{0}^{1} k(t, s) y(s) d s=x(t), \quad t \in[0,1], \\
y(0)=y_{0}=a,
\end{gathered}
$$

where $a$ is a given real number and $k(t, s) \in L^{2}([0,1) \times[0,1))$ and $x(t) \in L^{2}[0,1)$ are known functions for $t \in[0,1]$.

Lemma 1. For a polynomial in Bezier form

$$
y(t)=\sum_{i=0}^{n_{1}} a_{i, n_{1}} B_{i, n_{1}}(t)
$$

we have

$$
\frac{\sum_{i=0}^{n_{1}} a_{i, n_{1}}^{2}}{n_{1}+1} \geq \frac{\sum_{i=0}^{n_{1}+1} a_{i, n_{1}+1}^{2}}{n_{1}+2} \geq \cdots \geq \frac{\sum_{i=0}^{n_{1}+m_{1}} a_{i, n_{1}+m_{1}}^{2}}{n_{1}+m_{1}+1}
$$

where $a_{i, n_{1}+m_{1}}$ is the Bezier coefficient of $y(t)$ after being degreeelevated to degree $n_{1}+m_{1}$.

Proof. See [21]. 
The convergence of the approximate solution could be done in degree raising of the Bezier polynomial approximation.

Theorem 2. If the integral equation (7) has a unique $C^{1}$ continuous solution $\bar{y}$, then the approximate solution obtained by the control-point-based method converges to the exact solution $\bar{y}$ as the degree of the approximate solution tends to infinity.

Proof. Given an arbitrary small positive number $\epsilon>0$, by the Weierstrass Theorem (see [32]), one can easily find polynomial $Q_{1, N_{1}}(t)$ of degree $N_{1}$ such that $\left\|Q_{1, N_{1}}(t)-\bar{y}(t)\right\|_{\infty} \leq$ $\epsilon / 16$, where $\|\cdot\|_{\infty}$ stands for the $L_{\infty}$-norm over $[0,1]$. In particular, we have

$$
\left\|a-Q_{1, N_{1}}(0)\right\|_{\infty} \leq \frac{\epsilon}{16} .
$$

In general, $Q_{1, N_{1}}(t)$ does not satisfy the boundary conditions. After a small perturbation with constant polynomial $\alpha$, for $Q_{1, N_{1}}(t)$, we can obtain polynomial $P_{1, N_{1}}(t)=Q_{1, N_{1}}(t)+\alpha$ such that $P_{1, N_{1}}(t)$ satisfies the boundary condition $P_{1, N_{1}}(0)=$ $a$. Thus, $Q_{1, N_{1}}(0)+\alpha=a$. By using (10), one has

$$
\left\|a-Q_{1, N_{1}}(0)\right\|_{\infty}=\|\alpha\|_{\infty} \leq \frac{\epsilon}{16} .
$$

We have

$$
\begin{aligned}
\left\|P_{1, N_{1}}(t)-\bar{y}(t)\right\|_{\infty} & =\left\|Q_{1, N_{1}}(t)+\alpha-\bar{y}(t)\right\|_{\infty} \\
& \leq\left\|Q_{1, N_{1}}(t)-\bar{y}(t)\right\|_{\infty}+\|\alpha\|_{\infty} \leq \frac{\epsilon}{8}<\frac{\epsilon}{5} .
\end{aligned}
$$

Now, let

$$
\begin{aligned}
L P_{N}(t) & =L\left(t, P_{1, N_{1}}(t)\right) \\
& =P_{1, N_{1}}(t)-\int_{0}^{1} k(t, s) P_{1, N_{1}}(s) d s=x(t)
\end{aligned}
$$

for every $t \in[0,1]$. Thus, for $N \geq N_{1}$, one may find an upper bound for the following residual:

$$
\begin{aligned}
\left\|L P_{N}(t)-y(t)\right\|_{\infty} & =\left\|L\left(t, P_{1, N_{1}}(t)\right)-y(t)\right\|_{\infty} \\
\leq & \left\|P_{1, N_{1}}(t)-\bar{y}(t)\right\|_{\infty} \\
& +\int_{0}^{1}\left\|k(t, s) P_{1, N_{1}}(s) d s\right\|_{\infty} \\
\leq & C_{1}\left(\frac{\epsilon}{5}+\frac{\epsilon}{5}\right)<C_{1} \epsilon,
\end{aligned}
$$

where $C_{1}=1+\|k(t, s)\|_{\infty}$ is a constant.

Since the residual $R\left(P_{N}\right):=L P_{N}(t)-y(t)$ can be considered as a polynomial because if it is not a polynomial, we can use the Taylor series for it, we can represent the statement $R\left(P_{N}\right)$ by a Bezier form. Thus, we have

$$
R\left(P_{N}\right):=\sum_{i=0}^{m_{1}} d_{i, m_{1}} B_{i, m_{1}}(t) .
$$

Then, by Lemma 1, there exists an integer $M(\geq N)$ such that when $m_{1}>M$, we have

$$
\left|\frac{1}{m_{1}+1} \sum_{i=0}^{m_{1}} d_{i, m_{1}}^{2}-\int_{0}^{1}\left(R\left(P_{N}\right)\right)^{2} d t\right|<\epsilon
$$

which gives

$$
\frac{1}{m_{1}+1} \sum_{i=0}^{m_{1}} d_{i, m_{1}}^{2}<\epsilon+\int_{0}^{1}\left(R\left(P_{N}\right)\right)^{2} d t \leq \epsilon+C_{1}^{2} \epsilon^{2} .
$$

Suppose that $y(t)$ is an approximated solution of (7) obtained by the control-point-based method of degree $m_{2}\left(m_{2} \geq m_{1} \geq\right.$ $M)$. Let

$$
\begin{aligned}
R(t, y(t)) & =L(t, y(t))-y(t) \\
& =\sum_{i=0}^{m_{2}} c_{i, m_{2}} B_{i, m_{2}}(t), \quad m_{2} \geq m_{1} \geq M, t \in[0,1]
\end{aligned}
$$

Define the following norm for the difference-approximated solution $y(t)$ and the exact solution $\bar{y}(t)$ :

$$
\|y(t)-\bar{y}(t)\|:=\int_{0}^{1}|y(t)-\bar{y}(t)| d t .
$$

It is easy to show that

$$
\begin{aligned}
\|y(t)-\bar{y}(t)\| \leq & C(|y(0)-\bar{y}(0)| \\
& +\| R\left((t, y(t))-(t, \bar{y}(t)) \|_{2}^{2}\right. \\
= & C \int_{0}^{1} \sum_{i=0}^{m_{2}}\left(c_{i, m_{2}} B_{i, m_{2}}(t)\right)^{2} d t \\
\leq & \frac{C}{m_{2}+1} \sum_{i=0}^{m_{2}} c_{i, m_{2}}^{2} .
\end{aligned}
$$

The last inequality in (20) is obtained by Lemma 1 in which $C$ is a constant positive number. Now, by Lemma 1 and (15), it can be shown that

$$
\begin{aligned}
\|y(t)-\bar{y}(t)\| & \leq \frac{C}{m_{2}+1} \sum_{i=0}^{m_{2}} c_{i, m_{2}}^{2} \\
& \leq \frac{C}{m_{2}+1} \sum_{i=0}^{m_{2}} d_{i, m_{2}}^{2} \leq \frac{C}{m_{1}+1} \sum_{i=0}^{m_{1}} d_{i, m_{1}}^{2} \\
& \leq C\left(\epsilon+C_{1}^{2} \epsilon^{2}\right)=\epsilon_{1}, \quad m_{1} \geq M,
\end{aligned}
$$

where the last inequality in (21) is coming from (17).

Thus, from (21) we have

$$
\|y(t)-\bar{y}(t)\| \leq \epsilon_{1} .
$$

Since the infinite norm and the norm defined in (19) are equivalent, there is a $\rho_{1}>0$ where

$$
\|y(t)-\bar{y}(t)\|_{\infty} \leq \rho_{1} \epsilon_{1}=\epsilon_{2} .
$$

This completes the proof. 


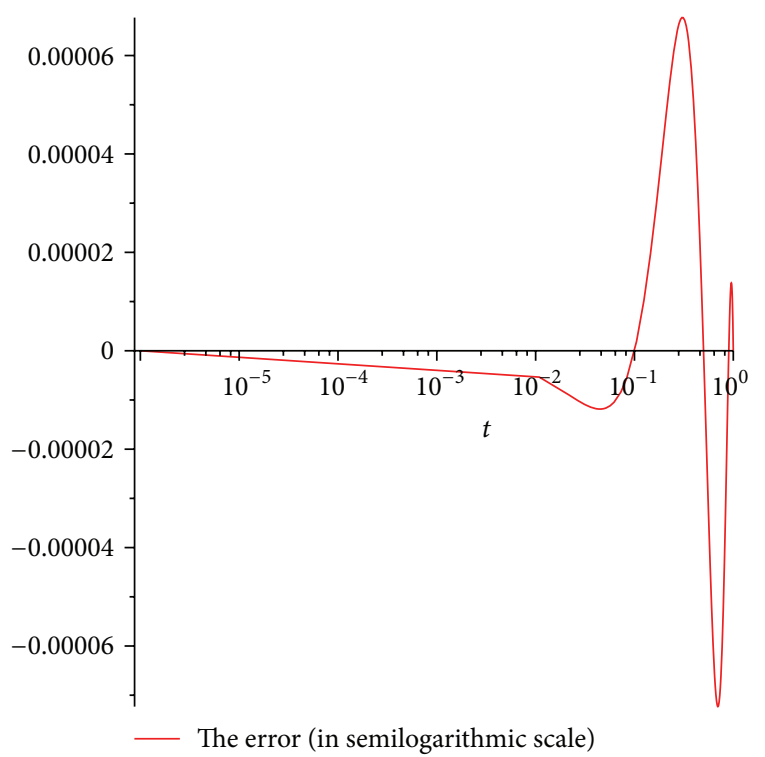

FIGURE 1: The error for Example 1.

\section{Numerical Examples}

In this Section, we present some test problems and apply the method presented in this paper for solving them. The wellknown symbolic software "Maple 12" has been employed for calculations.

Example 1. Consider the integral equation described by

$$
y(t)=\int_{0}^{1}(t+s) y(s) d s+e^{t}+(1-e) t-1, \quad y(0)=1
$$

For this integral equation, there exists the exact solution $y(t)=e^{t}$ (see [17]). With the method described in the paper, and with $n=4$ one can find the following approximate solution:

$$
\begin{aligned}
y(t)= & (1-t)^{4}+4.999424084 t(1-t)^{3} \\
& +9.506308758 t^{2}(1-t)^{2} \\
& +8.155525672 t^{3}(1-t)+2.718281828 t^{4}
\end{aligned}
$$

Figure 1 shows the value of error for Example 1 where the maximum error of hybrid Taylor and Block-Pulse functions [17] is $1.777834 \times 10^{-4}$ for $M=3$ and $N=20$.

Example 2. Consider the following integral equation:

$$
\begin{aligned}
y(t)= & \int_{0}^{1}\left(s^{2} t-\frac{3}{2} s t^{2}\right) y(s) d s+\frac{3}{4} t^{2} \\
& -\frac{4}{3} \operatorname{Ln}(2) t+\frac{5}{9} t+2 \operatorname{Ln}(t+1), \quad y(0)=0,
\end{aligned}
$$

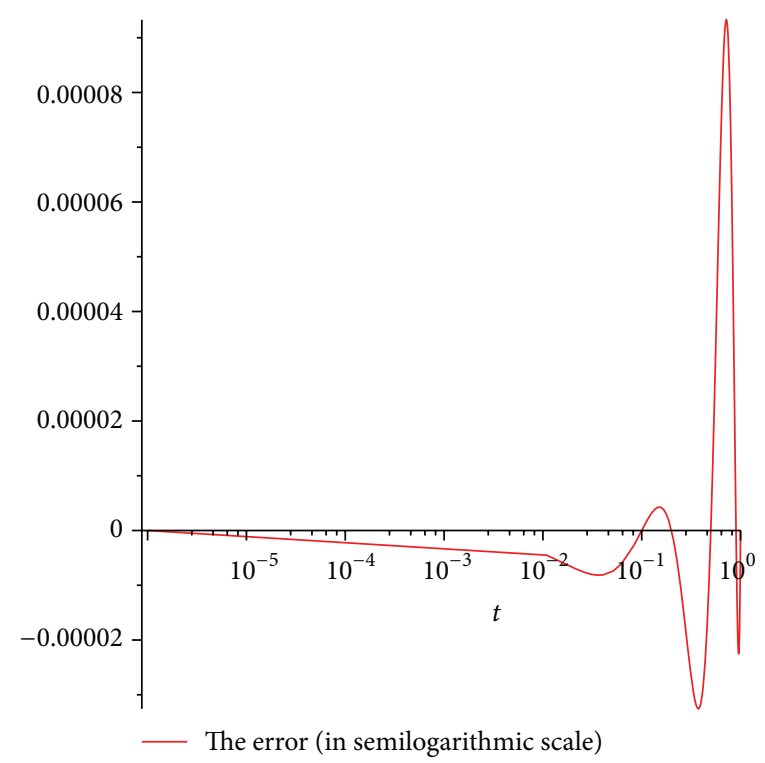

FIGURE 2: The error for Example 2.

$y(t)=2 \operatorname{Ln}(t+1)$, (see [17]). With the described method and with $n=5$, one can find the following solution:

$$
\begin{aligned}
y(t)= & 1.999482218 t(1-t)^{4}+7.008252137 t^{2}(1-t)^{3} \\
& +9.625408201 t^{3}(1-t)^{2}+5.93033 t^{4}(1-t) \\
& +1.386294361 t^{5} .
\end{aligned}
$$

The error curve of Example 2 is shown in Figure 2 where the maximum error of hybrid Taylor and Block-Pulse functions is $9.509965 \times 10^{-5}$ for $M=3$ and $N=80$ (see [17]). In Table 1, analytic, numerical results of the presented method, and the absolute error of the presented method are shown, respectively.

Example 3. Consider the following integral equation (see [17]):

$$
y(t)=-\frac{1}{3} \int_{0}^{1} e^{2 t-(5 / 3) s} y(s) d s+e^{2 t+(1 / 3)}, \quad y(0)=1,
$$

$y(t)=e^{2 t}$ (see [17]). With the described method and with $n=6$, one can find the following approximate solution:

$$
\begin{aligned}
y(t)= & (1-t)^{6}+7.998720114 t(1-t)^{5} \\
& +27.01533534 t^{2}(1-t)^{4} \\
& +49.29396712 t^{3}(1-t)^{3}+51.71626551 t^{4}(1-t)^{2} \\
& +29.55669277 t^{5}(1-t)+7.389056099 t^{6}
\end{aligned}
$$

Figure 3 shows the value of error for Example 3 where the maximum error of hybrid Taylor and Block-Pulse functions [17] is $4.625381 \times 10^{-5}$ for $M=4$ and $N=80$. 
TABLE 1: Exact and estimated values of $x(t)$ for Example 2.

\begin{tabular}{lccc}
\hline$t$ & Analytic $x(t)$ & Presented method & Errors of presented method \\
\hline 0.125 & 0.235566071312766 & 0.235569180133636 & $3.108820870 \times 10^{-6}$ \\
0.250 & 0.446287102628420 & 0.446276196803712 & $1.0905824708 \times 10^{-5}$ \\
0.375 & 0.636907462237070 & 0.636874894051300 & $3.2568185770 \times 10^{-5}$ \\
0.500 & 0.810930216216328 & 0.81093021615625 & $6.0078 \times 10^{-11}$ \\
0.625 & 0.971015631563402 & 0.971084086456756 & $6.8454893354 \times 10^{-5}$ \\
0.750 & 1.11923157587085 & 1.11932128616895 & $8.971029810 \times 10^{-5}$ \\
0.875 & 1.25721731884475 & 1.25723533320645 & $1.801436170 \times 10^{-5}$ \\
1.000 & 1.38629436111989 & 1.38629436100000 & $1.1989 \times 10^{-10}$ \\
\hline
\end{tabular}

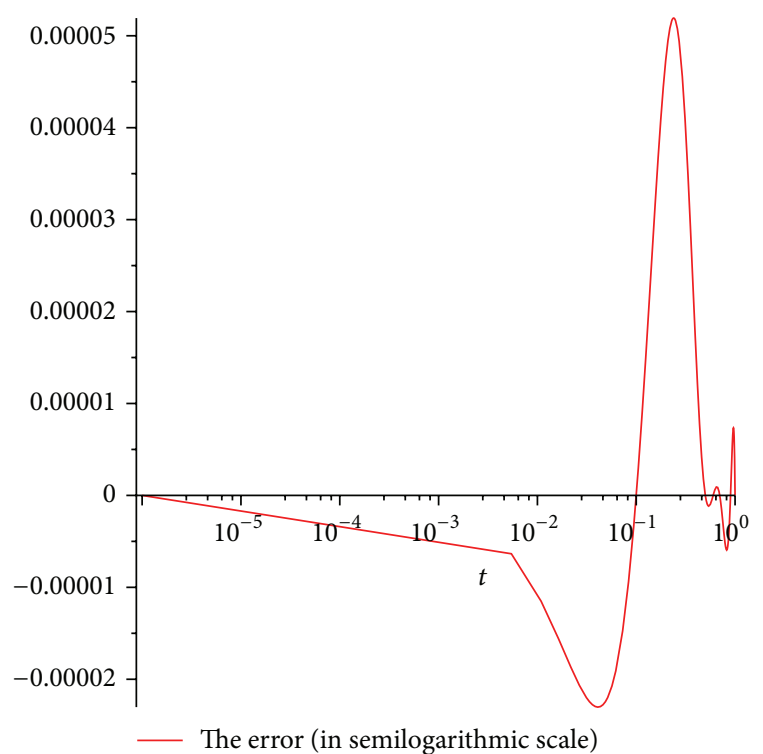

Figure 3: The error for Example 3.

Example 4. Consider the nonlinear Fredholm integral equation with exact solution $y(t)=\sinh (t)($ see [33]):

$$
\begin{array}{r}
y(t)=\sinh (t)-1+\int_{0}^{1}\left(\cosh \left(s^{2}\right)-y\left(s^{2}\right)\right) d s, \\
y(0)=0 .
\end{array}
$$

In our method, with $n=4$ one can find the following solution

$$
\begin{aligned}
y(t)= & 0.9995869312 t(1-t)^{3}+3.0045666 t^{2}(1-t)^{2} \\
& +3.158170163 t^{3}(1-t)+1.175201194 t^{4} .
\end{aligned}
$$

Figure 4 shows the value of error for Example 4.

\section{Conclusions}

A simple and effective algorithm based on Bezier curves is presented for solving Fredholm integral equations of the second kind. The method is computationally attractive and also reduces the CPU time and the computer memory while at the same time keeping the accuracy of the solution.

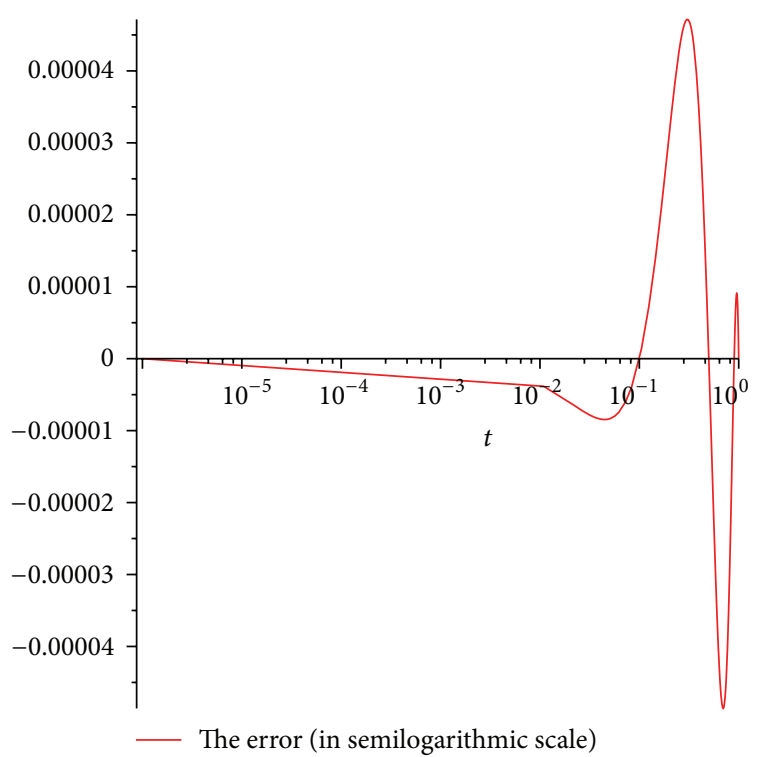

Figure 4: The error for Example 4.

\section{Conflict of Interests}

The authors declare that they have no conflict of interests regarding publication of this article.

\section{Acknowledgments}

The authors are very grateful to the referees for their valuable suggestions and comments that improved the paper. The third author acknowledges that this research was partially supported by the Universiti Putra Malaysia under the research Grant ERGS 1-2013(5527179).

\section{References}

[1] F. Bloom, "Asymptotic bounds for solutions to a system of damped integro-differential equations of electromagnetic theory," Journal of Mathematical Analysis and Applications, vol. 73, no. 2, pp. 524-542, 1980.

[2] K. Holmåker, "Global asymptotic stability for a stationary solution of a system of integro-differential equations describing the formation of liver zones," SIAM Journal on Mathematical Analysis, vol. 24, no. 1, pp. 116-128, 1993. 
[3] M. A. Abdou, "On a symptotic methods for Fredholm-Volterra integral equation of the second kind in contact problems," Journal of Computational and Applied Mathematics, vol. 154, no. 2, pp. 431-446, 2003.

[4] L. K. Forbes, S. Crozier, and D. M. Doddrell, "Calculating current densities and fields produced by shielded magnetic resonance imaging probes," SIAM Journal on Applied Mathematics, vol. 57, no. 2, pp. 401-425, 1997.

[5] A. D. Polyanin and A. V. Manzhirov, Handbook of Integral Equations, Chapman \& Hall/CRC Press, Boca Raton, Fla, USA, 2nd edition, 2008.

[6] M. A. Golberg, "The convergence of a collocation method for a class of Cauchy singular integral equations," Journal of Mathematical Analysis and Applications, vol. 100, no. 2, pp. 500$512,1984$.

[7] E. V. Kovalenko, "Some approximate methods for solving integral equations for mixed problems," Journal of Applied Mathematics and Mechanics, vol. 53, no. 1, pp. 85-92, 1989.

[8] B. I. Smetanin, "On an integral equation of axisymmetric problems for an elastic body containing an inclusion," Journal of Applied Mathematics and Mechanics, vol. 55, no. 3, pp. 371375, 1991.

[9] J. R. Willis and S. Nemat-Nasser, "Singular perturbation solution of a class of singular integral equations," Quarterly of Applied Mathematics, vol. 48, no. 4, pp. 741-753, 1990.

[10] J. I. Frankel, "A Galerkin solution to a regularized Cauchy singular integro-differential equation," Quarterly of Applied Mathematics, vol. 53, no. 2, pp. 245-258, 1995.

[11] W. F. Blyth, R. L. May, and P. Widyaningsih, "Volterra integral equations solved in Fredholm form using Walsh functions," The ANZIAM Journal, vol. 45, pp. C269-C282, 2004.

[12] M. H. Reihani and Z. Abadi, "Rationalized Haar functions method for solving Fredholm and Volterra integral equations," Journal of Computational and Applied Mathematics, vol. 200, no. 1, pp. 12-20, 2007.

[13] K. B. Datta and B. M. Mohan, Orthogonal Functions in Systems and Control, vol. 9, World Scientific, River Edge, NJ, USA, 1995.

[14] H. R. Marzban and M. Razzaghi, "Numerical solution of the controlled duffing oscillator by hybrid functions," Applied Mathematics and Computation, vol. 140, no. 2-3, pp. 179-190, 2003.

[15] M. Razzaghi and H.-R. Marzban, "A hybrid analysis direct method in the calculus of variations," International Journal of Computer Mathematics, vol. 75, no. 3, pp. 259-269, 2000.

[16] C.-H. Hsiao, "Hybrid function method for solving Fredholm and Volterra integral equations of the second kind," Journal of Computational and Applied Mathematics, vol. 230, no. 1, pp. 5968, 2009.

[17] K. Maleknejad and Y. Mahmoudi, "Numerical solution of linear Fredholm integral equation by using hybrid Taylor and blockpulse functions," Applied Mathematics and Computation, vol. 149, no. 3, pp. 799-806, 2004.

[18] M. Evrenosoglu and S. Somali, "Least squares methods for solving singularly perturbed two-point boundary value problems using Bézier control points," Applied Mathematics Letters, vol. 21, no. 10, pp. 1029-1032, 2008.

[19] K. Harada and E. Nakamae, "Application of the Bézier curve to data interpolation," Computer-Aided Design, vol. 14, no. 1, pp. 55-59, 1982.

[20] G. Nürnberger and F. Zeilfelder, "Developments in bivariate spline interpolation," Journal of Computational and Applied Mathematics, vol. 121, no. 1-2, pp. 125-152, 2000.
[21] J. Zheng, T. W. Sederberg, and R. W. Johnson, "Least squares methods for solving differential equations using Bézier control points," Applied Numerical Mathematics, vol. 48, no. 2, pp. 237$252,2004$.

[22] J. V. Beltran and J. Monterde, "Bézier solutions of the wave equation," in Computational Science and Its ApplicationsICCSA, vol. 3044 of Lecture Notes in Computer Science, pp. 631640, Springer, Berlin, Germany, 2004.

[23] R. Cholewa, A. J. Nowak, R. A. Bialecki, and L. C. Wrobel, "Cubic Bezier splines for BEM heat transfer analysis of the 2D continuous casting problems," Computational Mechanics, vol. 28, no. 3-4, pp. 282-290, 2002.

[24] C.-H. Chu, C. C. L. Wang, and C.-R. Tsai, "Computer aided geometric design of strip using developable Bézier patches," Computers in Industry, vol. 59, no. 6, pp. 601-611, 2008.

[25] A. T. Layton and M. Van de Panne, "A numerically efficient and stable algorithm for animating water waves," The Visual Computer, vol. 18, no. 1, pp. 41-53, 2002.

[26] J. Wu, "Least squares methods for solving partial differential equations by using Bézier control points," Applied Mathematics and Computation, vol. 219, no. 8, pp. 3655-3663, 2012.

[27] M. Gachpazan, "Solving of time varying quadratic optimal control problems by using Bézier control points," Computational \& Applied Mathematics, vol. 30, no. 2, pp. 367-379, 2011.

[28] F. Ghomanjani and M. H. Farahi, "The Bezier control points method for solving delay differential equation," Intelligent Control and Automation, vol. 3, no. 2, pp. 188-196, 2012.

[29] G. Farin, Curves and Surfaces for Computer Aided Geometric Design, Academic Press, New York, NY, USA, 1st edition, 1988.

[30] F. Ghomanjani, M. H. Farahi, and M. Gachpazan, "Bézier control points method to solve constrained quadratic optimal control of time varying linear systems," Computational \& Applied Mathematics, vol. 31, no. 3, pp. 433-456, 2012.

[31] Y. Q. Shi and H. Sun, Image and Video Compression for Multimedia Engineering, CRC Press, 2000.

[32] W. Rudin, Principles of Mathematical Analysis, McGraw-Hill, 1986.

[33] S. M. Mirzaei, "Homotopy perturbation method for solving the second kind of non-linear integral equations," International Mathematical Forum, vol. 5, no. 21-24, pp. 1149-1154, 2010. 


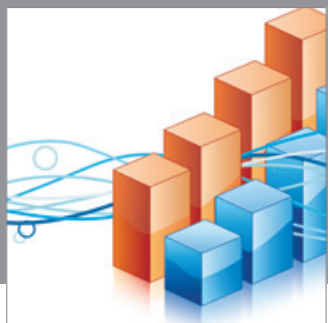

Advances in

Operations Research

mansans

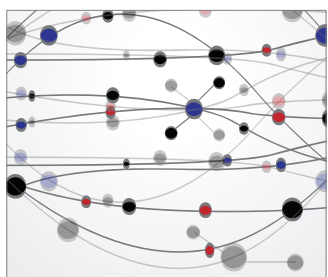

The Scientific World Journal
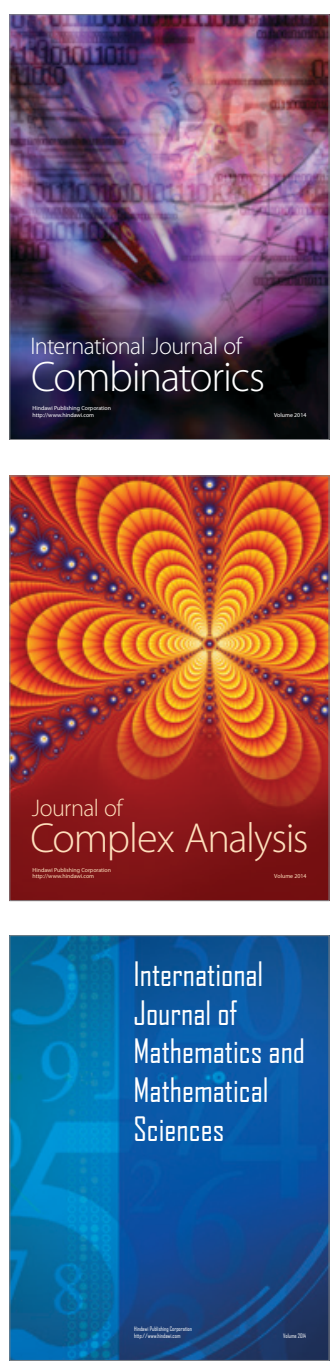
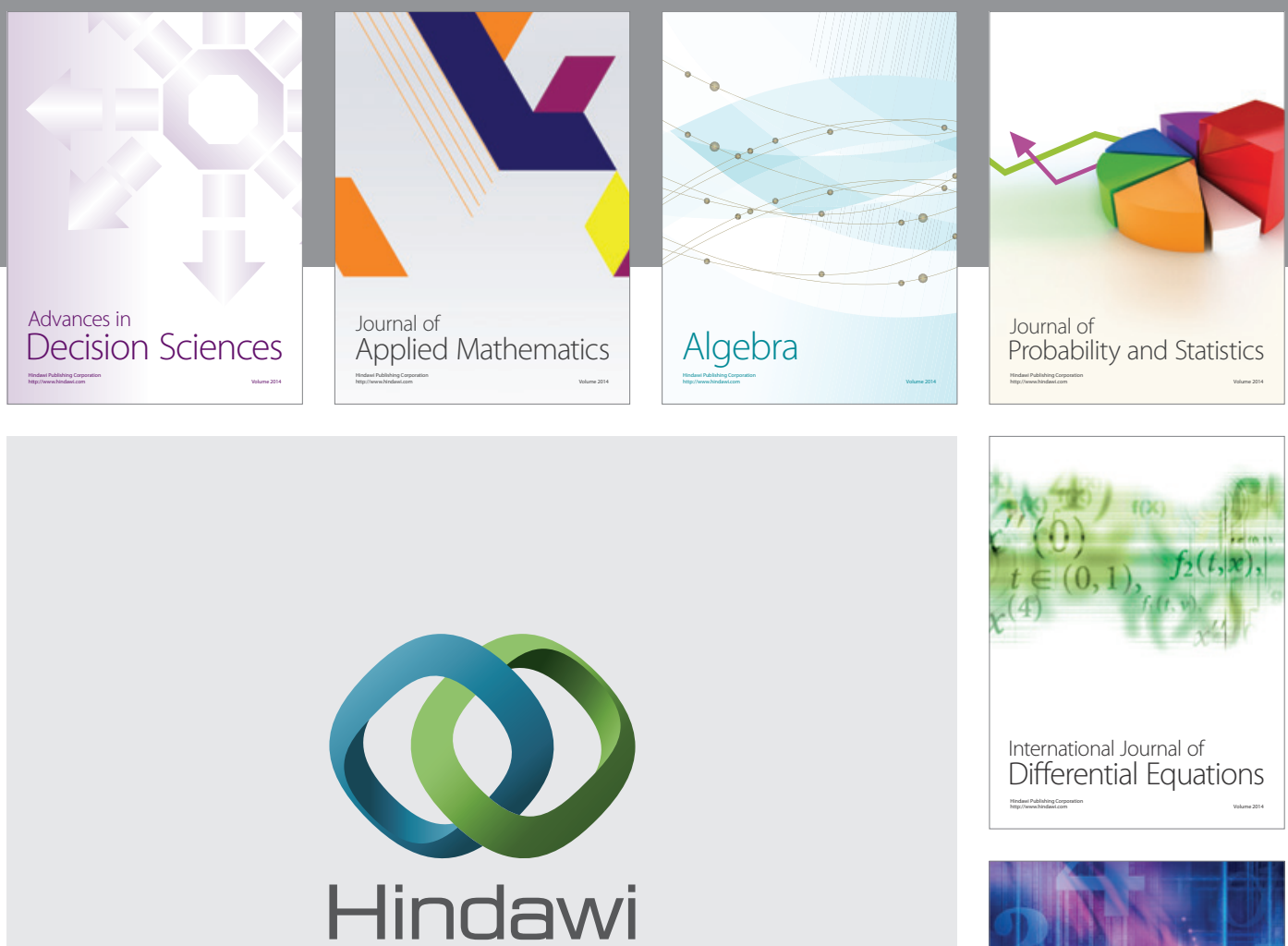

Submit your manuscripts at http://www.hindawi.com
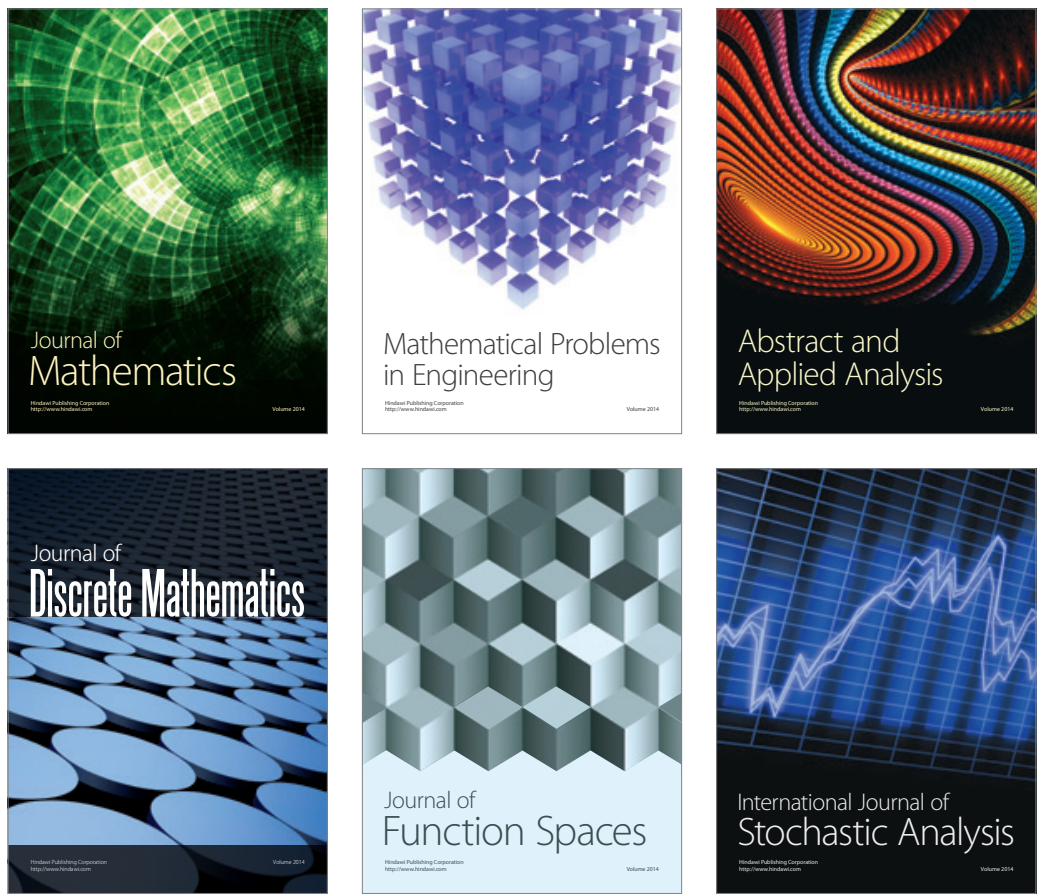

Journal of

Function Spaces

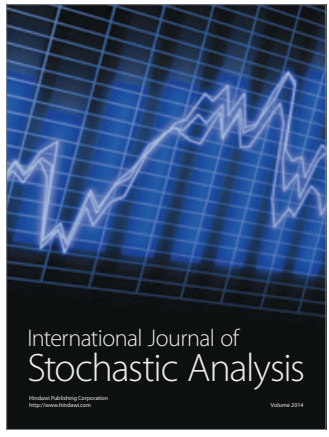

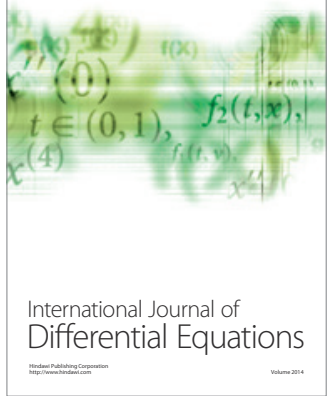
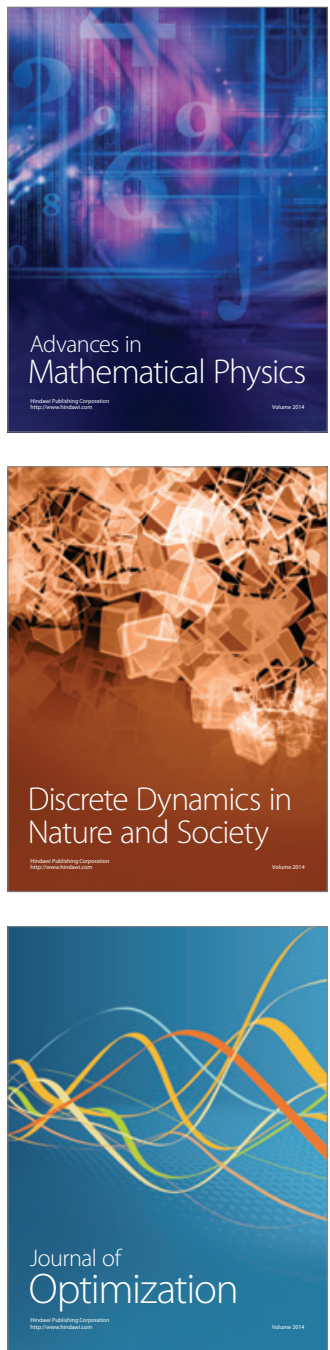